infection. On examination they were found to have hepatosplenomegaly, with normal cognition and development, Eye examination was normal, basal blood tests were normal as well as metabolic screen and cardiac Echo, CT chest showed interstitial changes, lysosomal enzyme screen showed marked deficiency of (ASM) Sphingomylinase activity and increased Chitotriosidase activity which is consistent with Nieman pick Type A/B disease. A blood sample has been sent for full gene sequencing of the Nieman Pick A/B gene. Currently they are well and thriving, with no major complications in the mean time

Discussion There is no specific treatment for NPD. Orthotopic liver transplantation in an infant with type A disease and cord blood transplantation in several type B NPD patients has been attempted with little or no success. BMT in a small number of type B NPD patients has been successful in reducing the spleen and liver volumes, the sphingomyelin content of the liver, the number of Niemann-Pick cells in the marrow, and radiologically detected infiltration of the lungs. ERT with recombinant human ASM is currently in clinical trials for the treatment of type B patients

Conclusion Niemann-Pick disease types A and B is estimated to affect 1 in 250,000 individuals, Chronic visceral ASMD could have a mild course with a relatively good outcome. Other factors such as environmental ones could contribute to the disease severity.

\section{P576 KERION: A NASTY SCALP INFECTION}

Ronan Callanan*, Foong Ying Wong, Emma Tierney, Bart Ramsay, Anne-Marie Murphy. UHL, Limerick, Ireland

\subsection{6/archdischild-2019-epa.910}

Background/Aims Kerion is a scalp condition that occurs in severe cases of scalp ringworm (tinea capitis) with the highest prevalence in children 3 to 10 years of age. It appears as an inflamed, thickened, pus-filled area, with scaly spots or patches of broken hair on the scalp. A kerion is treated with oral antifungal medicines because the fungus grows deep into the hair follicle where topical creams and lotions cannot penetrate. Oral corticosteroids are also started in cases where lesions are tender and to reduce the inflammation. Kerion is usually associated with infection by zoophilic dermatophytes, Tricophyton Verrucusom and Tricophyton Mentagrophyte, although other sources have been described. Our aim is to report an unusual scalp infection.

Methods The patient history, clinical presentation with photographs, examination and laboratory findings (skin scrapings were taken for mycology), treatment and outcome are described.

Results A previously well 6-year-old boy, of African origin, presented to the Paediatric Emergency Department with a 6month history of scalp infection. It initially started on the right temporal region and was treated with Canesten cream for 6 months. There was no improvement and the condition was treated with Flucloxacillin a week prior to presentation to us, at which point it had worsened and started spreading to the rest of his scalp. On examination there was a raised demarcated lesion on his right temporal area which appeared crusted and discharge was noted. There were also multiple round, dry, scaly patches on his scalp which were not infected. The dermatology team commenced him on oral
Itraconazole, Nizoral Shampoo and Paraffin Gel for 6 weeks, and oral steroids for 1 week.

Conclusion Kerion is not often seen in our paediatric department. This case raises awareness of a rarely seen condition in an Irish context.

\section{P577 JOINT MANIFESTATIONS AS A FORM OF ONSET IN CROHN'S DISEASE}

Radmila Elena Costachescu*, Corina Pienar, Liviu Pop. University of Medicine and Pharmacy 'Victor Babes', Timisoara, Romania

\subsection{6/archdischild-2019-epa.911}

Crohn's disease (CD) is an inflammatory bowel disease (IBD) of multifactorial etiology that affects all segments of the gastrointestinal tract and is associated with multiple extra-intestinal manifestations. Spondyloarthropathies are the most common extra-digestive manifestation of IBD's. Spondyloarthropathies primarily involve the axial skeleton, but mai also be associated with peripheral symptoms such as synovitis, dactylitis or enthesitis.

Objectives Case presentation of a teenager, aged 14, diagnosed with CD A1 L3L4B1p-score PCDAI 45, (November 2018) that presented with joint manifestations as a form of onset.

Case presentation The patient exhibits joint pain at the level of large joints of self-limiting character, as well as pain at the level of the costochondral joints associated with fever spikes. In recent history, over a period of two months, the patient presented up to 10 stools/day, without pathological features, with consecutive weight loss. An infectious etiology was suspected and empiric antibiotic treatment was instituted, with the evolution being unfavorable.

She is admitted to the clinic for further investigation and treatment. Biologically she presented: marked inflammatory syndrome, reactive thrombocytosis, hypochromic microcytic anemia, positive calprotectin. The suspicion of an IBD, is raised, multiple biopsies from the upper digestive tract being performed. The macro and microscopic appearance suggested Crohn's disease in the stage of active lesions. Induction therapy with exclusive enteral nutrition (EEN) is established, as well as azathioprine, to maintain remission. Evolution is favorable with the remission of joint symptoms in the first 2 weeks of EEN.

Conclusion In some cases, patients with Crohn's disease, due to chronic inflammatory syndrome, may develop symptoms similar to those encountered in spondyloarthropathies.

\section{P578 INTRAVENOUS MAGNESIUM SULPHATE INFUSION IN THE MANAGEMENT OF SEVERE TETANUS: A CASE SERIES}

${ }^{1}$ Norashikin Mohd Ranai ${ }^{*}$, Noor Shafina Mohd Nor, ${ }^{2}$ Foo Lai Sin, ${ }^{2}$ Jamaluddin Hj Mohamad. 'MARA University of Technology (UiTM), Selangor, Malaysia; ${ }^{2}$ Sungai Buloh Hospital, Selangor, Malaysia

\subsection{6/archdischild-2019-epa.912}

Background Tetanus is still prevalent in developing countries. In the management of tetanus, eradication of bacteria from the wound and neutralization of remaining toxins are detrimental. However, supportive management especially control of muscle spasm and airway protection is equally important. We 